\title{
Summary of workshops co-located with IE'09
}

\section{Introduction}

The 5th edition of the International Conference on Intelligent Environments was held in Castelldelfels (Catalonia, Spain), from July 19th to July 21st, 2009. The first day of the event hosted a number of workshops. This article provide an overview of these events.

\section{DOMe'09}

The 1st International Workshop on Digital Object Memories aimed to bring together technical experts, artists, designers, and potential end-users of Digital Object Memories to explore the technical, social, privacy, and legal implications of digital memory systems, to establish a common view on the underpinning requirements to Digital Object Memories, and to leverage cooperation in future activities.

Digital Object Memories comprise hardware and software components that physically and/or conceptually associate structured and unstructured digital information with real-world objects in an applicationindependent manner. If constantly updated, over time Digital Object Memories provide a meaningful record of an object's history and use. From a technical point of view, Digital Object Memories provide an open-loop infrastructure for the exchange of objectrelated information across application and environment boundaries, and as such allow for novel classes of applications in which rich object histories are created and exploited. From the user's point of view, Digital Object Memories create a new design space for everyday interactions and our relationship with physical artifacts. They could become sites for their owners' personal stories, but also afford people the opportunity to explore an object's provenance and connections to other elements of physical and digital life.

The workshop comprised paper presentations, an invited keynote talk, as well as plenary discussions between and after presentations. The first presentation session "Design Studies \& Applications" addressed topics like the use of physical objects as containers for personal experiences in home settings and museum exhibits, as well as on the use of digital product memories with the purpose of informing customers. The second presentation session comprised technical presentations related to the implementation of smart object systems with digital memory functionality. Relevant topics included structures for organizing and storing memory content, the acquisitions of object memory information through sensors in the environment, and the detection of usage patterns based on object memory information. The keynote talk was given by Wolfgang Maass and addressed the topic of object memories in the context of smart products, which use object memories in order to realize added-value services to the manufacturer, reseller, and consumer.

Discussions revealed several interesting questions related to the implementation and application of digital object memory systems, including the question of authoring (automatically vs. manually, and by whom), nature and kind of memories (physical, social, cultural), ethical aspects (ownership, etc.), the role of the container object with respect to memory content and ownership of the memory and the object, and the semantic and representation/interpretation of object memory content (potentially out of its original context).

To promote creative thinking about how Digital Object Memories could transform our experience of the world around us, a design study award was announced by the organizers. Professional artists \& designers, researchers, and art \& design students were encouraged to submit summaries of design concepts, that will challenge the research community to consider broad landscape of potential applications of Digital Object Memories. The award was won by Daniela Petrelli and Ann Light for their contribution "Memory Baubles and History Tinsels".

The organizers thank all participants (see Fig. 1) for making DOMe'09 an interesting and worthwhile event 


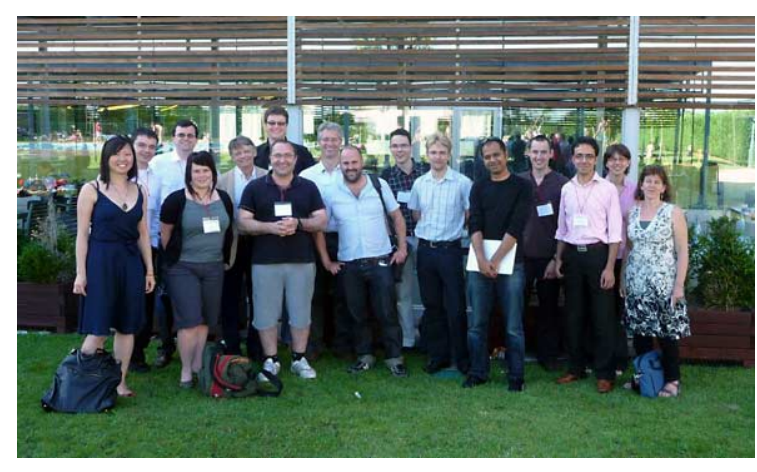

Fig. 1. Participants of DOMe'09.

with inspiring presentations and outstanding lively and fruitful discussions. A follow-up workshop is planned for 2010. Further information on DOMe'09 and future workshops can be found at the workshop website www.dfki.de/dome-workshop/.

\section{Michael Schneider, Alexander Kröner, Patrick Olivier, and Peter Stephan \\ (co-chairs of DOMe'09)}

\section{RFID'09}

The 1st International Workshop on RFID Technology: Concepts, Practices and Solutions (RFID’09) has been held at Castelldelfels, Spain on July 19, 2009 prior to the 5th edition of the International Conference on Intelligent Environments. More than 15 participants attended this workshop for which 11 presentations have been selected by the international program committee out of 16 submissions.

The opening ceremonies were chaired by $\mathrm{PhD}$. Julio C. Encinas. After the official opening, Dr. Andrés García Higuera from University of Castilla La Mancha gave a keynote talk on "RFID: Lies and true". Prof. García is the president of the Spanish Society of Traceability. In his very interesting talk, he presented very interesting material related to concepts and technical information of RFID technology. Highlighting some lies that some people tell us about how it works RFID. This triggered intensive discussions about how to explore the limits of RFID in order to have an unavoidable advance of this technology in many fields.

Two technical sessions complemented the program. The first session chaired by Andrés García Higuera (University of Castilla La Mancha) on "Automation of production and Logistics" discussed the issues of adaptability of RFID in production and logistics areas.
It has been discussed how a traceability system based on RFID that will work in a slaughterhouse guarantee the quality requirements established by the CRDO (Consejo Regulador de la Denominación de Origen or the governing board of the protected designation of origin). Another presentation showed an experimental platform which is a test-bench in order to development new management methods with the application of RFID System and to examine and quantify the impact of these management methods on distribution centers. Another important issue is the improvement of the warehouse operations management using real time RFID Information. Another talk present a three-level based RFID System. With the aim of improve the process of identification and monitoring of condition of products in different levels of the Supply Chain. For doing this, passive and active tags, and the necessary RFID readers are connected using ZigBee networks and GPRS modules.

Another hot research topic has been discussed in the first afternoon session on "RFID Applications" chaired by Julio C. Encinas (Autonomous University of Baja California). Many RFID applications and services are currently being developed. Because of this the paper presentations were quite diverse and all interesting. One interesting issue was the analysis of a particular case of study consisting of a full pallet with multiple UHF tags readings using a reader and following the EPC Class1 Generation 2 standard. In order to register the Received Strength Signal Intensity (RSSI) and the ratio of tags read and tag readings. Another important issue is the use of Web Services-based prototype in order to obtain data over de internet using RFID. The following presentation presented several prototypes using RFID and wireless communications. In order to convert tangible items into an Internet applications. The fourth talk addressed the issue of the deployment of services in medical environments that can be carried out with RFID/NFC and how to apply security measures in them. Finally, the last presentation shows us the importance of Customer Relationship Management (CRM) and how to enhance using a knowledge-based framework incorporating RFID-generated data.

The workshop ended with a roundtable session discussed the different topics presented in the workshop, especially, the key note presentation. The speech of Andres Garcia inspired us to have as a concluding remark that RFID is a very powerful technology and offer important opportunities for business and society. But, some people don't know the reach of RFID and offer to the customers more then RFID can do, cre- 


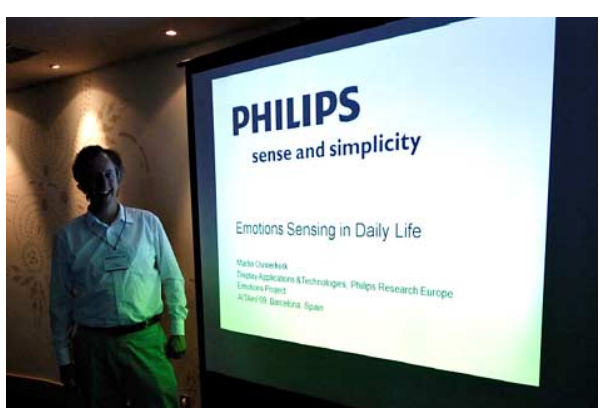

Fig. 2. Keynote Speaker.

ating false expectative about this technology. The 1st workshop of the RFID Technology: Concepts, Practices and Solutions (RFID'09) has been success in particular when considering the quality of the presentations. This could only be achieved due to the excellent work of authors and reviewers.

\section{Julio C. Encinas and Andrés García Higuera (co-chairs of RFID'09)}

\section{AITAmI'09}

The 4th International Workshop on Artificial Intelligence Techniques for Ambient Intelligence continues a series of successful events started in 2006 and aimed at considering the application of traditional and new AI-based solutions to the area of Ambient Intelligence.

The event included the presentation of papers, posters, a keynote and a discussion session. This edition was opened by Dr. Martin Ouwerkerk (Philips) whose keynote on: "Emotions Sensing in Daily Life" (see Fig. 2) gave the audience an overview on the state of the art of sensing emotions in a human being to improve the performance of an intelligent environment. This presentation also highlighted the difficulties of reliably sensing emotions, the costs involved, issues of acceptability as well as the important benefits that can be obtained from it.

A special session organized by A. Sgorbissa, F. Mastrogiovanni and R. Zaccaria, "Mobile Robots in Automated Building Systems", was focused on the use of and interaction amongst robots and Intelligent Environments. Issues covered here were focused on the difference of approaches amongst traditional robotics and robotics designed for a specific environment. One bottleneck for the effective realization of such environments populated by "robotic appliances" is the lack of standards and low interoperability.

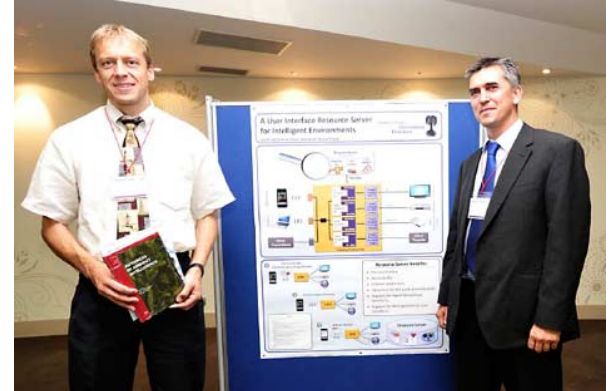

Fig. 3. Best poster presentation award.

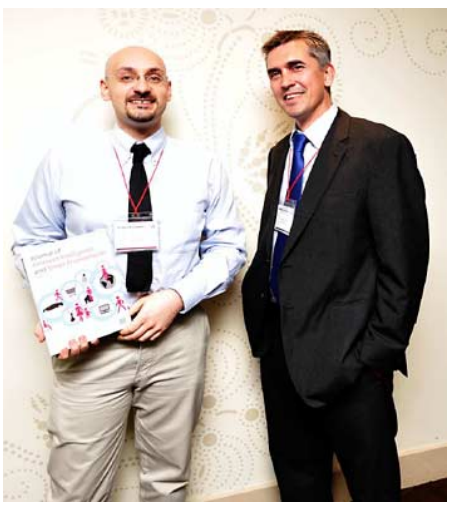

Fig. 4. Best paper presentation award.

Another special session organized by J. O'Donoghue, "Intelligent Environments Supporting Healthcare and Well-being", focused on the use of intelligent environments as places were humans can be cared and encouraged to improve or sustain a healthy lifestyle. Some of the issues raised in this session were the design principles that: ideally these systems should be deployed before a big decline of elderly person starts (so that the system learns smoothly from the very beginning and notice abnormal changes) and that "humans should ask and machines should follow".

The best presented poster and best presented paper were selected by voting amongst the attendees and the prizes were awarded (respectively) to: "Why We Need a User Interface Resource Server for Intelligent Environments" by G. Zimmermann (see Fig. 3), and "Support for Context-aware Monitoring in Home Healthcare" by A. Mileo, D. Merico, and R. Bisiani (see Fig. 4).

Some shared concerns amongst the audience were: how services can be efficiently (quickly and unobtrusively) composed, how to personalize a system to different humans, and the acceptability of technology (how the system should know when to act and when 
not to!). These topics relate to the overall focus for this edition and particularly to Martin Ouwerkerk's keynote. Given the importance of the topic and its complexity, this will probably be a central issue for several editions to come.

The organizers wish to acknowledge the participation of the attendees and warmly invites the readers of this article to the next edition of the event which will be co-located with IE'2010.

Juan Carlos Augusto and Diane J. Cook (co-chairs of AITAmI'09)

\section{EDAmI'09}

The First Workshop on Ethical Design of Ambient Intelligence (EDAmI'09) presented research and studies that have tackled with issues related to new technologies, ethics and user experience of products, applications and services that are based on vision of ambient intelligence. Another goal of the workshop was to focus on ethical guidelines that have been produced especially for the design of new technologies. Since Ambient Intelligence applications, based on information and communication technology, are embedded in our environment and everyday objects clearly raise several ethical issues it was indeed very timely to have a workshop dedicated to this issue. While the technical design of smart environments is still quite challenging it seems that it could be even more challenging to do social design in this very broad area of research and development. The Workshop was organised by ETICA (http://www.etica-project.eu) and MinAmi (http://www.fp6-minami.org/) projects, both of which are tackling with ethics and new technologies on practical and theoretical levels. The papers of the workshop offered good state of the art view to the ethical design of ambient intelligence while some of the papers had a quite focused look at the issue, with others handling the theme in a more theoretical manner.

We had six papers looking at different angles of ethics of ambient intelligence in our workshop. Papers by Satinder P. Gill, Ethics of Ambience, by Alessia Santuccio, Marco Marabelli, Penny Duquenoy, Philippe Goujon, Sylvain Lavelle and Norberto Patrignani, Moving Towards an Ethical Governance of New Emerging Technologies and by Bernd C.Stahl, Simon Rogerson and Kutoma J. Wakunuma, Understanding Ethical Issues of Emerging AmI Technologies in Europe (A Framework), reviewed issue in more abstract level, while papers by by Michel Arnaud, Privacy, security and safety in e-ticketing, by Steven Dorrestijn, Design and Ethics of Product Impact on User Behavior and Use Practices and by Veikko Ikonen, Eija Kaasinen and Marketta Niemelä, Defining Ethical Guidelines for Ambient Intelligence Applications on a Mobile Phone, had affiliated ethical dimensions to the design of forthcoming applications and services sketched in particular projects.

The main issues brought out in discussion after presentations and in general discussion in the end of the workshop handled things like:

1. Is ethical design possible? Whose ethics, which groundings?

2. How to involve various technology developers and other relevant stakeholders to the discussion and design from ethical perspective.

3. Guidelines or recommendations based on ethical thinking which may be either to general or too focused for the wider usage. How to accomplish ethical design tool that could work on different levels in design.

4. Ethicality of design as usability attributes.

In workshop discussions we realised that there work on these themes which is currently going on in many areas as well as dedicated workshops and conferences (e.g. ETHICOMP, ETICA, ICT that makes the difference) so it seems that interest in the theme has evolved positively. Although it now seems that there is a growing interest in the theme of ethical design, the big challenge still seems to be the governance of the ethical design in the area of ambient intelligence. In that respect it seems that we will need to continue our work in the next EDAmI workshop 2010.

Veikko Ikonen, Catherine Flick, Philippe Goujon, Eija Kaasinen, Bernd Stahl and Kutoma Wakunuma (co-chairs of EDAmI'09)

\section{SOOW'09}

The 1st International Workshop on Smart Offices and Other Workplaces (SOOW 09) was organized by Prof. Peter Mikulecký and Dr. Pavel Cech, both from the University of Hradec Kralove, Czech Republic, in co-operation with Prof. Carlos Ramos, director of the GECAD, and coordinator professor at the Polytechnic of PortoŠs Institute of Engineering (ISEP), Por- 
tugal. The area of smart offices and other workplaces of this kind has naturally its technical point of view, which was covered as one of most important topics of the workshop. However, the workshop aimed also to cover much broader but related topics, as organizational and personal motivations for managers in using smart workplaces; specific types and models of smart workplaces for various applications; ubiquity and distributed nature of smart workplaces; or user modelling in smart workplaces, to mention just a few of the topics.

The event included the presentation of papers, short papers (instead of posters), a keynote delivered by Prof. Carlos Ramos and a discussion session.

The keynote was presented by Professor Carlos Ramos under the title "Cognitive Robots or Ambient Intelligence?". It has covered an interesting comparison of differences and connections between cognitive robotics and ambient intelligence areas with a number of illustrating examples.

Further on, the programme of this half-of-a-day workshop included five full paper presentations and two short presentations of accepted posters. The full papers were presented by Carlos Freitas (IGAMSAI Idea Generation with Autonomous Multimedia Gathering for Meetings Summaries in AmI), Adrien Joly
(Workspace Awareness without Overload: Contextual Filtering of Social Interactions), Peter Mikulecký (Notes on Smart Workplaces), Carlos Ramos (Adapting Information Displays Contents in Smart Spaces according User Emotions and Personality), and Pavel Cech (The Proposal of Intelligent Assistant for Graduation Thesis Preparation). The two short presentation were delivered by Connor Muldoon (Exercise in the Smart Workplace) and Margarida Romero (Time Awareness Tool for Enhancing Group TimesŠ Coordination in the Virtual Workspace).

The presented papers highlighted the need for further effort to be made to appropriately represent contextual information and provide for its semantic processing. The conclusions of the final discussion stressed that the area of smart offices and other similar workplaces goes behind technical solutions. It is necessary to focus the attention also on social issues, psychological aspects, as well as on specific application areas. The organizers and participants of the SOOW 09 workshop acknowledged very much the possibility of organizing the workshop with this focus and expressed the belief in continuing with this workshop series.

Peter Mikulecký, Pavel Cech, and Carlos Ramos (co-chairs of SOOW'09) 